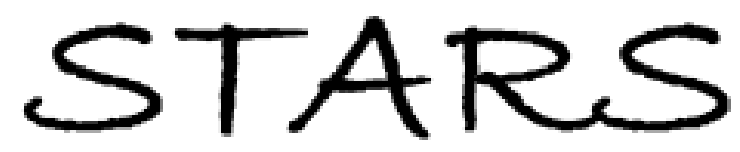

University of Central Florida

STARS

$1-1-2006$

\title{
Biodiversity of sessile and motile macrofauna on intertidal oyster reefs in Mosquito Lagoon, Florida
}

Michelle L. Boudreaux

Jennifer L. Stiner

Linda J. Walters

University of Central Florida

Find similar works at: https://stars.library.ucf.edu/facultybib2000

University of Central Florida Libraries http://library.ucf.edu

This Article is brought to you for free and open access by the Faculty Bibliography at STARS. It has been accepted for inclusion in Faculty Bibliography 2000s by an authorized administrator of STARS. For more information, please contactSTARS@ucf.edu.

\section{Recommended Citation}

Boudreaux, Michelle L.; Stiner, Jennifer L.; and Walters, Linda J., "Biodiversity of sessile and motile macrofauna on intertidal oyster reefs in Mosquito Lagoon, Florida" (2006). Faculty Bibliography 2000 s. 5972.

https://stars.library.ucf.edu/facultybib2000/5972

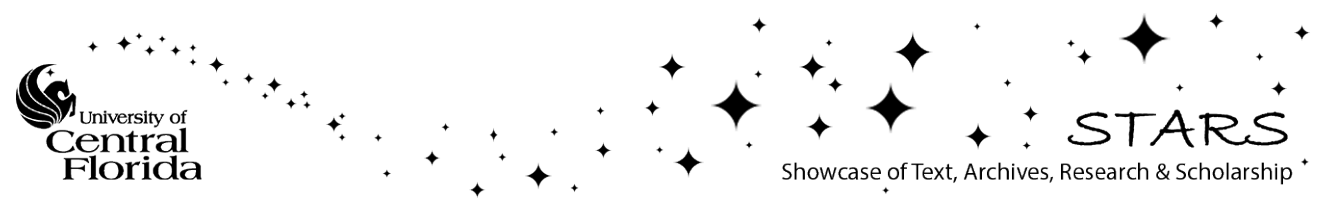




\title{
BioOne COMPLETE
}

\section{BIODIVERSITY OF SESSILE AND MOTILE MACROFAUNA ON INTERTIDAL OYSTER REEFS IN MOSQUITO LAGOON, FLORIDA}

\author{
Authors: MICHELLE L. BOUDREAUX, JENNIFER L. STINER, and LINDA J. WALTERS \\ Source: Journal of Shellfish Research, 25(3) : 1079-1089 \\ Published By: National Shellfisheries Association \\ URL: https://doi.org/10.2983/0730-8000(2006)25[1079:BOSAMM]2.0.CO;2
}

BioOne Complete (complete.BioOne.org) is a full-text database of 200 subscribed and open-access titles in the biological, ecological, and environmental sciences published by nonprofit societies, associations, museums, institutions, and presses.

Your use of this PDF, the BioOne Complete website, and all posted and associated content indicates your acceptance of BioOne's Terms of Use, available at www.bioone.org/terms-of-use.

Usage of BioOne Complete content is strictly limited to personal, educational, and non-commercial use. Commercial inquiries or rights and permissions requests should be directed to the individual publisher as copyright holder.

BioOne sees sustainable scholarly publishing as an inherently collaborative enterprise connecting authors, nonprofit publishers, academic institutions, research libraries, and research funders in the common goal of maximizing access to critical research. 


\title{
BIODIVERSITY OF SESSILE AND MOTILE MACROFAUNA ON INTERTIDAL OYSTER REEFS IN MOSQUITO LAGOON, FLORIDA
}

\author{
MICHELLE L. BOUDREAUX, ${ }^{1 *}$ JENNIFER L. STINER ${ }^{2}$ AND LINDA J. WALTERS \\ Department of Biology, University of Central Florida, 4000 Central Florida Boulevard, Orlando, \\ Florida 32816; ' Everglades Partners Joint Venture, 701 San Marco Blvd. Suite 1201, Jacksonville, \\ Florida 32207; ${ }^{2}$ BCI Engineers \& Scientists, 2000 E. Edgewood Drive Suite \# 215, \\ Lakeland, Florida 33803
}

\begin{abstract}
Our research focused on determining the diversity and abundance of sessile and motile macrofauna that use intertidal reefs of the eastern oyster Crassostrea virginica for feeding, settlement space or refuge in Mosquito Lagoon, Florida. Five replicate lift nets were deployed at six sites (three impacted reefs with seaward margins of disarticulated shells, three reference reefs without dead margins) to determine the species composition and numbers present on these reef types. All nets were deployed intertidally on backreef areas on living oyster reefs, just above mean low water. One and a half liters of live oysters and oyster shells were placed in each net $\left(1 \mathrm{~m}^{2}\right)$ on deployment. Nets were surveyed for all fauna monthly for one year. Metrics used to evaluate habitat use were species richness (total number of different species found) and density (total number of organisms per net). Comparisons were also made between community assemblages found on the two different types of reefs in the area (with and without dead margins) and for sessile species, recruitment on living oysters versus disarticulated shells. Forty sessile and 64 motile species of macroorganisms were found utilizing the oyster reefs in Mosquito Lagoon. However, recruitment on live oysters was twice that on disarticulated shells. Significant temporal variations were documented. When the two reef types were compared, however, no differences were found.
\end{abstract}

KEY WORDS: oysters, Crassostrea virginica, habitat use, fishes, decapods, barnacles, invertebrates, lift nets

\section{INTRODUCTION}

Human activities threaten the productivity, diversity, and survival of coastal resources, leading to a growing need to understand and manage all coastal zones (e.g., Jackson et al. 2001). The Indian River Lagoon system (IRL) on the east central Florida coast is one such place. This estuary extends $251 \mathrm{~km}$, from Ponce de Leon Inlet to Jupiter Inlet. The Lagoon system is a series of three distinct, but connected, estuaries: the Indian River, the Banana River and Mosquito Lagoon. This lagoon system may contain the richest biota of any estuary in North America (Provancha et al. 1992). It supports over 3,000 animal and plant species, 50 of which are listed as threatened or endangered. Commercially important intertidal reefs of the eastern oyster Crassostrea virginica are common in this estuarine system.

Diversity is extremely high in the IRL because of its location within a zoogeographic transition zone (e.g., Walters et al. 2001, Smithsonian Institution 2006). Researchers have documented the substantial species diversity of many habitats and taxa in IRL waters: seagrass and its associated organisms (e.g., Virnstein et al. 1983, Dawes et al. 1995); finfish (Gilmore 1977, Gilmore 1995, Tremain \& Adams 1995); elasmobranchs (Snelson \& Williams 1981) and decapods (Smithsonian Institution 2006). To date, there have been no studies of the biodiversity on intertidal oyster reefs in the IRL.

Three-dimensional reef structures of Crassostrea virginica are created by years of successive settlement of larvae on adult shells (Dame 1996). Through its structural complexity, these ecosystem engineers create heterogeneity that is rare in marine systems dominated by soft-bottom habitats (e.g., Bartol et al. 1999, Micheli \& Peterson 1999). Organisms use oyster reefs for many different reasons; mobile species may: (1) feed directly on live oysters, (2) use shell surfaces for spawning and (3) seek refuge from predation

*Corresponding author. E-mail: mLboudreaux03@hotmail.com within oyster clusters (e.g., Tolley \& Volety 2005), whereas sessile species use oyster reefs for attachment space.

Previous studies on intertidal oyster reef biodiversity include: Wells 1961 (North Carolina), Dame 1979 (South Carolina), Bahr and Lanier 1981 (south Atlantic coast), Crabtree and Dean 1982 (South Carolina), Wenner et al. 1996 (South Carolina), Coen et al. 1999a (South Carolina), Posey et al. 1999 (North Carolina), O'Beirn et al. 2004 (Virginia) and Tolley et al. 2005 (Florida, Gulf of Mexico). In most of these studies, the primary focus was on motile species (fish and crustaceans). Our study adds to this database by investigating the recruitment of motile macrofauna on backreef regions of intertidal oyster reefs of Crassostrea virginica in the IRL system along the Atlantic Coast of Florida. In addition, this is the first study in Florida to quantify diversity and abundance of all sessile macrofauna on oyster reefs.

\section{METHODS}

\section{Study Site}

All research was conducted in Mosquito Lagoon, within the boundaries of Canaveral National Seashore $\left(28^{\circ} 90.68 \mathrm{~W}\right.$; $80^{\circ} 82.06 \mathrm{~N}$ ) (Fig. 1). Except where dredged, the average depth of the Lagoon is less than $1 \mathrm{~m}$ and the current is primarily winddriven (Walters et al. 2001). Annual salinity ranges between 18 and 45 ppt, depending on rainfall (Grizzle 1990, Walters et al. 2001).

Within a 5-y period (1998-2003), the number of recreational boat registrations within the counties that border Mosquito Lagoon increased by $43 \%$ (Wall et al. 2005). This increasing intensity of year-round boating has helped create piles of disarticulated shells (dead margins) on the seaward edges of oyster reefs along major navigational channels in these shallow waters (Grizzle et al. 2002, Wall et al. 2005). We compared back-reef areas on reefs with and without dead margins to determine if recreational boating pressures influenced biodiversity. 


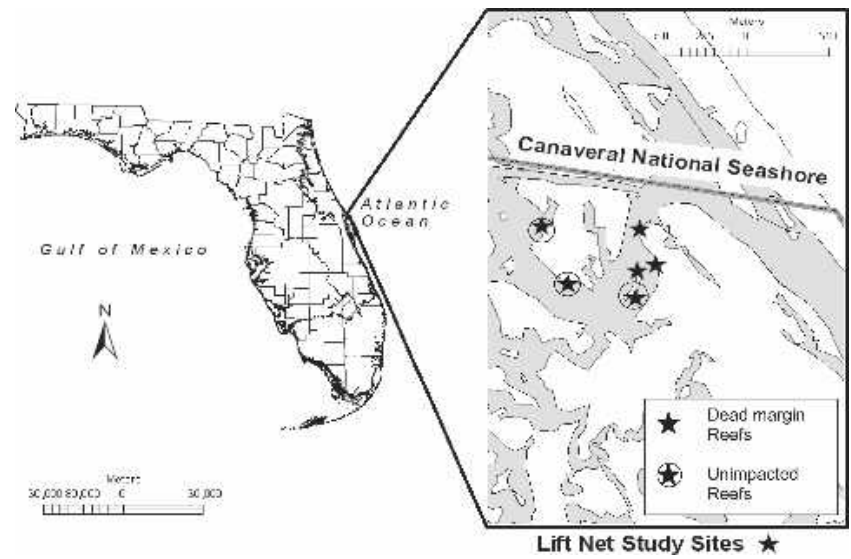

Figure 1. Lift nets study sites in Mosquito Lagoon, Florida.

\section{Lift Net Field Sampling}

Six oyster reefs were selected for this study, three impacted reefs (with dead margins) and three reference reefs (without dead margins). All were within a 5-km radius (Fig. 1). Five replicate lift nets were placed on the back-reef area of each reef. The protected back-reef areas were chosen to minimize the loss of nets caused by water motion.

Lift net methods were adapted from Crabtree and Dean (1982), Coen et al. (1996a), and later modified by Tolley et al. (2005) for use in Florida systems. We further modified the protocol to include the enumeration of sessile species recruiting to oyster reefs. Lift net frames were $1 \mathrm{~m}^{2}$ and created from $3.8 \mathrm{~cm}$ diameter PVC. The nets were $0.5 \mathrm{~m}$ deep. The sides of the nets were made from $3.2 \mathrm{~cm}$ diameter opening mesh and the bottom was made from a $1-\mathrm{m}$ square of $0.2-\mathrm{cm}$ diameter opening mesh. The two mesh sizes were machine-sewed together using extra strength cloth thread. The sewn mesh was attached to the PVC frame with cable ties (tensile strength: $11 \mathrm{~kg}$ ).

Lift nets were deployed intertidally, just above mean low water, on living oyster reefs. Volume normalized oysters and oyster shells in good condition $(1.5 \mathrm{~L})$ were placed in the lift nets. Half $(0.75 \mathrm{~L})$ were single, disarticulated shells from adults (Mean \pm SE length: $77.5 \pm 1.4 \mathrm{~mm}$; weight: $21.5 \pm 1.1 \mathrm{~g}$ ) and half were similar-sized live clusters collected from the oyster reef. All were mechanically scraped clean of epiflora and epifauna. New shells and clusters were placed into the nets each month. Additionally, at the time of net retrieval, all nets were cleaned to remove organisms that had settled on the mesh or PVC frames.

Lift nets were retrieved by swiftly picking up the nets on two sides and collecting all recruited motile and sessile organisms. In the laboratory, we identified all organisms within $24 \mathrm{~h}$ and returned them alive to Mosquito Lagoon. Only sessile organisms attached to oyster shells within the lift nets were counted. Nets were collected monthly for 12 mo (June 2004 to July 2005). No data was collected for September 2004 because Hurricanes Charley, Jeanne and Frances required removal of nets and prevented data collection. Specimens of each species were preserved in $70 \%$ isopropanol to create a species archive for the University of Central Florida.

\section{Environmental Variables}

Permanent temperature monitors (Onset Stowaway Tidbit Temperature Loggers) were attached to cinder blocks and de- ployed at each site in water at the same depth as the lift nets. Temperature data were collected once each hour. Salinity was measured on net retrieval using a portable refractometer. Three sediment traps were deployed at each site at the same depth as the lift nets to determine sediment load accumulations during the 4-wk intervals between sampling. Each replicate, cylindrical PVC pipe sediment trap $(10-\mathrm{cm}$ diameter $\times 25 \mathrm{~cm}$ deep) was submerged flush with the substrate (Lenihan 1999). We capped traps underwater at the time of retrieval. The sediment traps were retrieved concurrently with the lift nets and new traps were immediately deployed to replace them. Total sediment mass was determined by drying samples at $60^{\circ} \mathrm{C}$ for $48 \mathrm{~h}$ in a drying oven (Econotherm Model Number 51,221,126) and weighing contents on a toploading balance (O'Haus Scout 2-Model Number SC6010). Relative grain size was determined by grinding the dried sediment and sorting samples with a sieve $(0.062 \mathrm{~mm})$ to separate the silt/clay from the sand/gravel fractions.

\section{Analyses}

For all cases where analysis of variance (ANOVA) tests were run, prior to running the ANOVAs, homogeneity of variance and normality were tested using Levene and Kolmagorov-Smirnov tests. If significant differences were found with ANOVA, post-hoc Tukey-Kramer tests were run. Data assumptions of variance and normality were met for all ANOVAs at the $P=0.05$ level, thus the data were not transformed.

\section{Sessile Macrofauna}

Response variables of species richness (total number of different species) and density (total number of individuals) were analyzed using a 4-way, nested. ANOVA. The factors in the nested ANOVAs were: (1) reef type (reefs with dead margins or reference reefs), (2) month, (3) site and (4) shell type (disarticulated shells or live oysters in clusters). Reef type, month and shell type were fixed factors, whereas site was random. Shell type was nested within site, and site was nested within reef type.

\section{Motile Macrofauna}

Community metrics of motile species were similarly examined with a 3-way ANOVA. Response variables of species richness and density were examined as in sessile species. For each ANOVA, the factors were reef type (fixed), site nested within reef type (random), and month (fixed).

\section{Sediment Loads}

A 3-way ANOVA was conducted to test whether sediment loads on oyster reefs varied as a function of the following fixed factors: reef type (reference or dead margins) and month. The third factor, site, was random and nested within reef type.

\section{RESULTS}

\section{Biodiversity and Composition}

\section{Sessile Macrofauna}

Twenty-five species of sessile invertebrates recruited to oysters and oyster shells in the lift nets during our study (Table 1). Barnacles in the genus Balanus (Arthropoda) dominated all samples numerically. Tube worms in the genus Hydroides, the jingle shell Anomia simplex, the eastern slipper shell Crepidula astrasolea, 
TABLE 1.

Total numbers of sessile species collected in lift nets on intertidal oyster reefs in Mosquito Lagoon, Florida.

\begin{tabular}{|c|c|c|c|c|c|c|c|c|c|c|c|c|c|c|c|}
\hline Phylum & Species & $\begin{array}{l}\text { Common } \\
\text { Name }\end{array}$ & Total & $6 / 04$ & $7 / 04$ & $8 / 04$ & $10 / 04$ & $11 / 04$ & $12 / 04$ & $1 / 05$ & $2 / 05$ & $3 / 05$ & $4 / 05$ & $5 / 05$ & $6 / 05$ \\
\hline \multirow[t]{3}{*}{ Porifera } & $\begin{array}{l}\text { Hymeniacidon } \\
\text { heliophila }\end{array}$ & Sun sponge & 28 & 2 & 2 & 1 & 0 & 11 & 0 & 3 & 0 & 1 & 2 & 4 & 2 \\
\hline & $\begin{array}{r}\text { Halichondria } \\
\text { melandocia }\end{array}$ & $\begin{array}{l}\text { Black volcano } \\
\text { sponge }\end{array}$ & 66 & 1 & 0 & 0 & 0 & 0 & 5 & 0 & 0 & 0 & 0 & 3 & 57 \\
\hline & Cliona spp. & Boring sponge & 13 & 0 & 1 & 0 & 0 & 0 & 0 & 9 & 1 & 2 & 0 & 0 & 0 \\
\hline \multirow[t]{2}{*}{ Cnidaria } & Aiptasia pallida & Sea anemone & 2 & 0 & 0 & 0 & 0 & 0 & 0 & 2 & 0 & 0 & 0 & 0 & 0 \\
\hline & $\begin{array}{l}\text { Haliplanella } \\
\text { luciae }\end{array}$ & Striped anemone & 2 & 0 & 0 & 0 & 0 & 0 & 0 & 0 & 0 & 1 & 1 & 0 & 0 \\
\hline \multirow[t]{2}{*}{ Annelida } & Hydroides spp. & Tube worms & 2842 & 658 & 491 & 760 & 250 & 134 & 111 & 66 & 33 & 65 & 55 & 42 & 177 \\
\hline & Sabella spp. & $\begin{array}{l}\text { Feather duster } \\
\text { worm }\end{array}$ & 46 & 2 & 1 & 9 & 6 & 4 & 6 & 7 & 0 & 0 & 1 & 9 & 1 \\
\hline \multirow[t]{2}{*}{ Arthropoda } & $\begin{array}{l}\text { Balanus } \\
\text { eburneus }\end{array}$ & Ivory barnacle & 8782 & 2070 & 2447 & 1380 & 720 & 430 & 210 & 145 & 87 & 132 & 99 & 251 & 811 \\
\hline & $\begin{array}{l}\text { Balanus } \\
\text { amphitrite }\end{array}$ & $\begin{array}{l}\text { Purple striped } \\
\text { barnacle }\end{array}$ & 1524 & 438 & 461 & 450 & 46 & 16 & 13 & 9 & 1 & 5 & 6 & 8 & 71 \\
\hline \multirow[t]{11}{*}{ Mollusca } & $\begin{array}{l}\text { Crassostrea } \\
\quad \text { virginica recruits }\end{array}$ & Eastern oyster & 722 & 173 & 92 & 54 & 69 & 104 & 91 & 7 & 3 & 18 & 4 & 12 & 95 \\
\hline & Anomia simplex & Jingle shell & 1120 & 186 & 178 & 184 & 151 & 93 & 83 & 41 & 4 & 43 & 45 & 80 & 32 \\
\hline & $\begin{array}{l}\text { Crepidula } \\
\quad \text { astrasolea }\end{array}$ & $\begin{array}{l}\text { Eastern slipper } \\
\text { shell }\end{array}$ & 1178 & 287 & 154 & 207 & 149 & 69 & 59 & 31 & 4 & 25 & 44 & 73 & 76 \\
\hline & $\begin{array}{l}\text { Crepidula } \\
\quad \text { fornicata }\end{array}$ & $\begin{array}{l}\text { Atlantic slipper } \\
\text { shell }\end{array}$ & 40 & 12 & 0 & 3 & 4 & 4 & 0 & 9 & 0 & 1 & 1 & 0 & 6 \\
\hline & Diodora cayensis & Keyhole limpet & 3 & 1 & 1 & 1 & 0 & 0 & 0 & 0 & 0 & 0 & 0 & 0 & 00 \\
\hline & Atrina rigida & Pen shell & 1 & 0 & 0 & 0 & 0 & 0 & 1 & 0 & 0 & 0 & 0 & 0 & 0 \\
\hline & Tagelus divisus & Jacknife clam & 1 & 0 & 0 & 0 & 0 & 0 & 0 & 0 & 1 & 0 & 0 & 0 & 0 \\
\hline & $\begin{array}{l}\text { Brachidonetes } \\
\text { exuctus }\end{array}$ & Scorched mussel & 4 & 0 & 0 & 2 & 0 & 0 & 1 & 0 & 1 & 0 & 0 & 0 & 0 \\
\hline & Geukensia demissa & Ribbed mussel & 128 & 21 & 20 & 19 & 7 & 6 & 4 & 7 & 0 & 2 & 4 & 19 & 18 \\
\hline & Mytella charruana & Charru mussel & 3 & 0 & 0 & 0 & 3 & 0 & 0 & 0 & 0 & 0 & 0 & 0 & 0 \\
\hline & $\begin{array}{l}\text { Lithophaga } \\
\text { bisulcata }\end{array}$ & $\begin{array}{l}\text { Mahogany } \\
\text { date mussel }\end{array}$ & 1 & 0 & 0 & 0 & 0 & 0 & 0 & 0 & 0 & 0 & 0 & 0 & 1 \\
\hline \multirow[t]{4}{*}{ Ectoprocta } & Bugula neritina & $\begin{array}{l}\text { Common } \\
\text { bryozoan }\end{array}$ & 195 & 1 & 1 & 0 & 0 & 2 & 0 & 16 & 0 & 18 & 82 & 48 & 27 \\
\hline & $\begin{array}{l}\text { Hippoporina } \\
\text { verrilli }\end{array}$ & Lacy bryozoan & 40 & 0 & 0 & 1 & 28 & 11 & 0 & 0 & 0 & 0 & 0 & 0 & 0 \\
\hline & $\begin{array}{l}\text { Zoobotryon } \\
\text { verticillatum }\end{array}$ & Spaghetti bryozoan & 2 & 0 & 0 & 0 & 0 & 0 & 0 & 0 & 0 & 0 & 0 & 0 & 2 \\
\hline & Perophera viridis & Encrusting ascidian & 16 & 0 & 2 & 0 & 1 & 5 & 3 & 3 & 0 & 2 & 0 & 0 & 0 \\
\hline Chordata & Styela plicata & Rough sea squirt & 87 & 0 & 0 & 0 & 26 & 17 & 2 & 1 & 0 & 1 & 15 & 6 & 19 \\
\hline
\end{tabular}

and the eastern oyster Crassostrea virginica were also very abundant (Table 1). Mollusca represented the most abundant phyla, with nine species found. Other phyla represented included Annelida, Cnidara, Porifera, Ectoprocta and Chordata (Table 1). Outside of the lift nets, 15 additional species of sessile organisms were found in small numbers on the intertidal oyster reefs and nearby subtidal areas throughout the course of our study in Mosquito Lagoon, although they do not represent any additional phyla (Table 2).

Measures of oyster community metrics with sessile invertebrates exhibited clear trends in Mosquito Lagoon. Species richness and the density differed temporally, because of the month of sampling (ANOVA: $P<0.001$; Fig. 2, 3; Table 3, Table 4). Richness was significantly higher during June, July, August and October $(P$ $<0.001$; Fig. 2). Additionally, February had the lowest richness (Fig. 2). Density, the number of organisms per net, was significantly higher in June, July and August of 2004 than all other sampling periods (ANOVA; $P<0.001$; Fig. 3). Furthermore, spe- cies richness and density were higher on living oysters in clusters than on single disarticulated oyster shells (ANOVA for both: $P<$ 0.001; Tables 3, 4; Fig 4). Species found only on live oysters included mussel Lithophaga bisulcata, acsidian Perophera virdis and bryozoan Hippoprina verrilli. Reef type (reefs with dead margins or reference reefs) did not have a significant influence on the community metrics, species richness $(P=0.098)$ or density $(P=$ 0.207 ) (Tables 3,4). Site did not have a significant effect on species richness (ANOVA: $P=0.964)$ or density $(P=0.644)$ (Tables 3, 4).

\section{Motile Macrofauna}

During this study, 64 motile species were found on oyster reefs in Mosquito Lagoon. Fifty-one species were collected using lift nets (Table 5) and an additional 13 species were observed by researchers elsewhere on reefs and in nearby subtidal waters (Table 2). Chordata was the most abundant phyla found to be 
TABLE 2.

Additional macrofauna observed on oyster reefs within Mosquito Lagoon. These species were not collected in the lift nets.

\begin{tabular}{|c|c|c|}
\hline $\begin{array}{l}\text { Sessile Species } \\
\text { Phylum }\end{array}$ & Species Name & Common Name \\
\hline Annelida & Polydora websteri & Oyster mud worm \\
\hline \multirow[t]{6}{*}{ Mollusca } & Modiolus americans & Tulip mussel \\
\hline & Mercenaria mercenaria & Hard shelled clam \\
\hline & Anadara transversa & Tranverse ark \\
\hline & Anadara ovalis & Blood ark \\
\hline & Martesia cuneiformis & Striated wood paddock \\
\hline & Crepidula convexa & Convex slipper shell \\
\hline \multirow[t]{2}{*}{ Ectoprocta } & Conopeum spp. & Lacy crust bryozoan \\
\hline & $\begin{array}{l}\text { Zoobotryon verticillatum } \\
\text { Hippoporina verrilli }\end{array}$ & Moss bryozoan \\
\hline \multirow[t]{4}{*}{ Chordata } & Mogula manhattensis & Sea grape \\
\hline & Botrylloides nigrum & Black tunicate \\
\hline & Botryllus planus & Royal tunicate \\
\hline & $\begin{array}{l}\text { Botrylloides schlosseri } \\
\text { Didemnum sp. }\end{array}$ & Goldenstar tunicate \\
\hline \multicolumn{3}{|l|}{ Motile species } \\
\hline Phylum & Species Name & Common Name \\
\hline \multirow[t]{4}{*}{ Arthropoda } & Hexapanopeus angustifrons & Narrow mud crab \\
\hline & Limulus polyphemus & Horseshoe crab \\
\hline & Neopanope sayi & Say's mud crab \\
\hline & Pinnotheres ostreum & Oyster pea crab \\
\hline \multirow[t]{8}{*}{ Mollusca } & Aplysia brasiliana & Sooty sea hare \\
\hline & Busycon contrarium & Lightening whelk \\
\hline & Busycon spiratum & Pear whelk \\
\hline & Fasciolaria hunteria & Banded tulip \\
\hline & Fasciolaria tulipa & True tulip \\
\hline & Melongena corona & Crown conch \\
\hline & Pleuroploca gigantean & Florida horse conch \\
\hline & Polinices duplicatus & Atlantic moon snail \\
\hline Chordata & Symphurus plagiusa & Blackcheek tonguefish \\
\hline
\end{tabular}

utilizing the oyster reefs, with 23 fish species found. Mollusca were the second most prevalent phyla, with 20 different species found (Tables 2, 5). Other phyla that were represented in the collections included: Arthropoda (18 species), Echinodermata (2 species) and Annelida (1 species) (Tables 2, 5). The bigclaw snapping shrimp Alpheus heterochaelis and the flat mud crab Eurypanopeus depressus, dominated the collections numerically yearround (Table 5).

Species richness and density differed because of the month of

TABLE 3.

Four-factor nested ANOVA comparing species richness of sessile organisms in lift nets. Factors were reef type (dead margin or reference; fixed), shell type (live clusters or disarticulated shells) nested within site nested within reef type (random), and month (fixed).

\begin{tabular}{lrrrc}
\hline \hline \multicolumn{1}{c}{ Source } & df & $\begin{array}{c}\text { Mean } \\
\text { Square }\end{array}$ & \multicolumn{1}{c}{ F } & Significance \\
\hline Reef type & 1 & 64.201 & 4.625 & 0.098 \\
Site (Reef type) & 4 & 13.881 & 0.133 & 0.964 \\
Shell type (Site (Reef type)) & 6 & 104.321 & 42.236 & $<0.001$ \\
Month & 11 & 85.401 & 34.576 & $<0.001$ \\
Residual & 697 & & & \\
\hline
\end{tabular}

TABLE 4.

Four-factor nested ANOVA comparing density of sessile organisms in lift nets. Factors were reef type (dead margin or reference; fixed), shell type (live clusters or disarticulated shells) nested within site nested within reef type (random), and month (fixed).

\begin{tabular}{lrcrc}
\hline \hline \multicolumn{1}{c}{ Source } & df & $\begin{array}{c}\text { Mean } \\
\text { Square }\end{array}$ & \multicolumn{1}{c}{ F } & Significance \\
\hline Reef type & 1 & 68406.006 & 2.265 & 0.207 \\
Site (Reef type) & 4 & 30195.728 & 0.657 & 0.644 \\
Shell type (Site (Reef type)) & 6 & 45976.603 & 16.335 & $<0.001$ \\
Month & 11 & 35020.837 & 12.443 & $<0.001$ \\
Residual & 697 & & & \\
\hline
\end{tabular}

sampling (ANOVA: both $P<0.001$; Table 6, Table 7). Richness was higher in November December, January and May (Fig. 2). Density was significantly higher in June, November and December 2004 than any of the other sampling dates (Fig. 3). Reef type (reefs with dead margins or reference reefs) did not have a significant influence on species richness (ANOVA: $P=0.985$ ) or density $(P=0.624)$ (Tables 6,7$)$. Site did not significantly affect species richness (ANOVA: $P=0.181$ ), however it did significantly affect density $(P=0.002)$ (Tables 6,7$)$.

\section{Environmental Variables}

During the 13-mo study, the monthly mean temperatures in Mosquito Lagoon ranged from $16^{\circ} \mathrm{C}$ to $31^{\circ} \mathrm{C}$ (Fig. 5a). Salinity ranged from 25-35 ppt (Fig. 5b), falling within the typical average range of 25-45 ppt for the monthly mean in Mosquito Lagoon (Walters et al. 2001). The lowest salinity (25 ppt) occurred immediately after the 2004 hurricane season (Fig. 5b). Total sediment loads differed significantly between sites (ANOVA: $P=0.011$ ), but not reef type $(P=0.234)$ (Table 8 ; Fig. 5 c). After sediment loads were separated into fractions, percent silt/clay still did not differ significantly between reef type (ANOVA: $P=0.454$ ) or sites $(P=0.482)$ (Table 9; Fig. 5d). During the months of June 2004 and June 2005, both sediment load and percent silt/clay differed temporally (ANOVA: $P=0.004$ and $<0.001$, respectively). Tukey results showed sediment loads to peak in June 2004,

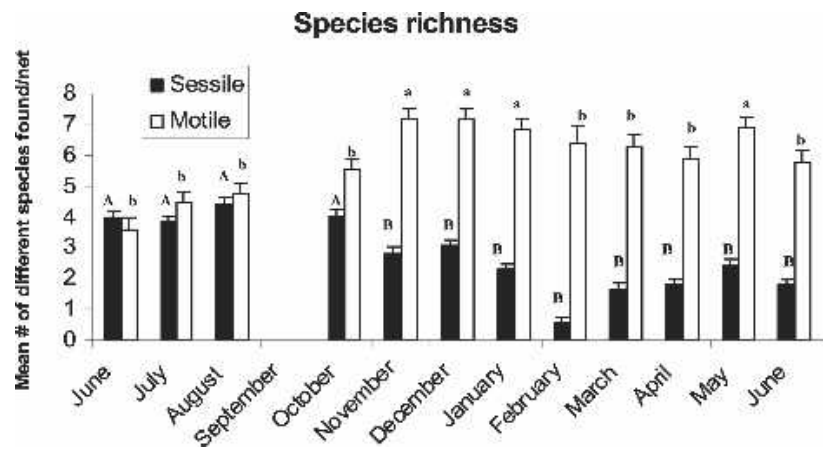

Figure 2. Monthly mean richness (total number of species) \pm SE per net per month from June 2004 to June 2005. September data are missing because of 2004 hurricane activity. When compared with ANOVA and a Tukey-Kramer post-hoc test, significantly higher months are depicted by $A$ versus $B$ for sessile species and a versus $b$ for motile species. 


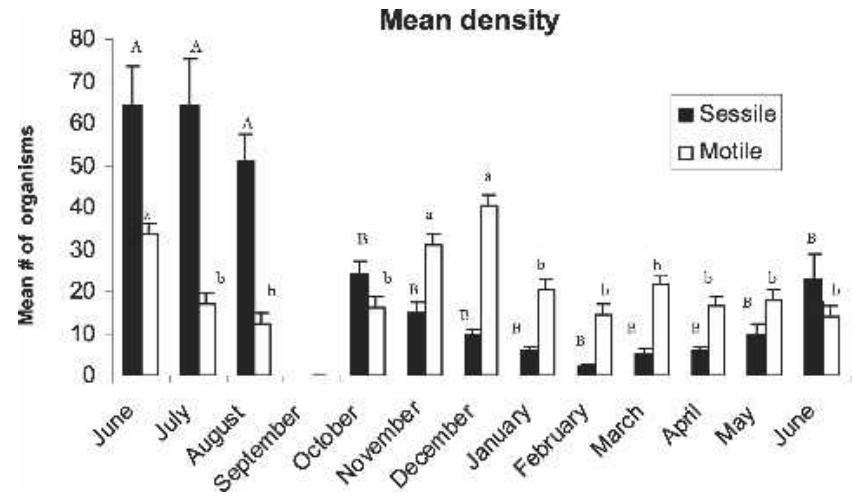

Figure 3. Monthly mean density (number of individuals) \pm SE per net per month from June 2004 to June 2005. September data missing because of 2004 hurricane activity. When compared with ANOVA and a Tukey-Kramer post-hoc test, significant higher months are depicted by $A$ versus B for sessile species and a versus b for motile species.

whereas percent silt/clay fractions were highest in June and July 2004, and January 2005 (Fig. 5c, 5d).

\section{DISCUSSION}

The assemblage of marcofauna associated with the intertidal oyster reefs during our lift net study was similar to those previously reported on oyster reefs in the southeastern United States (sessile species: Wells 1961; motile species: Meyer 1994, Breitburg 1999, Coen et al. 1999, Posey et al. 1999, Glancy et al. 2003,
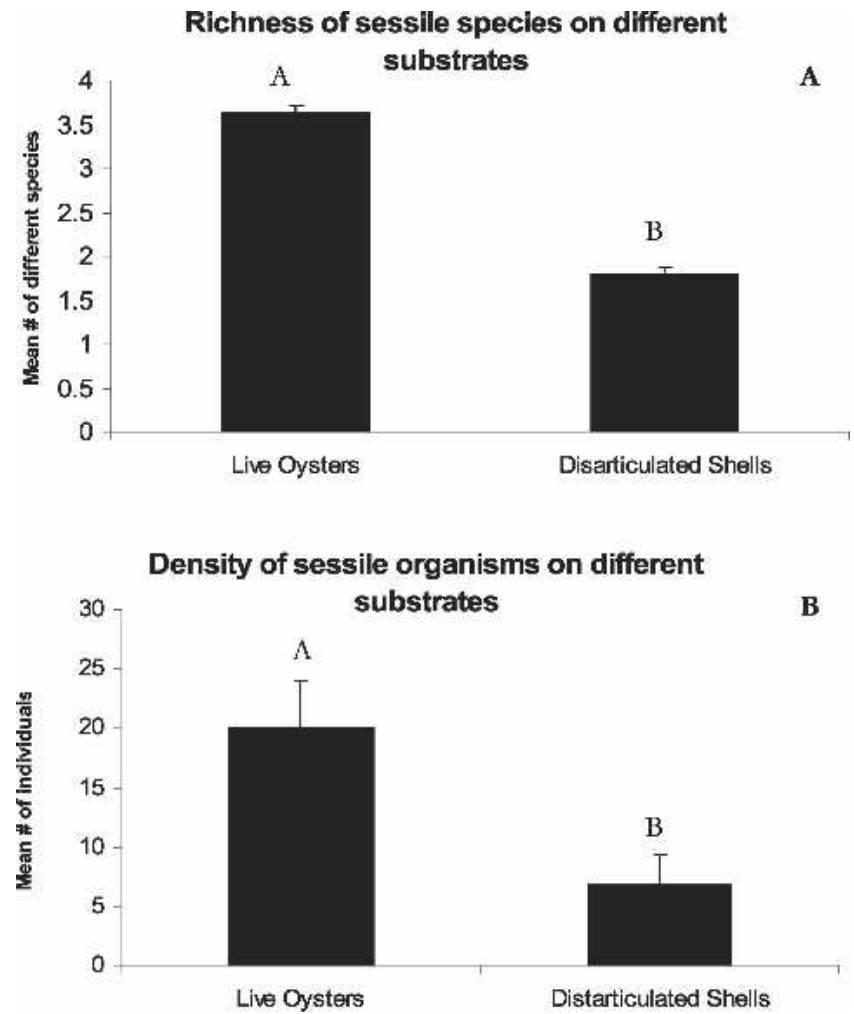

Figure 4. (a) Mean richness ( $\pm \mathrm{SE}$ ) of sessile organisms on live oysters compared with disarticulated shells. (b) Mean density $( \pm \mathrm{SE})$ of sessile organisms on live oysters compared with disarticulated shells. Significant differences are depicted by $\mathbf{A}$ versus $\mathbf{B}$.
TABLE 6.

Three-factor nested ANOVA comparing species richness of motile species in lift nets. Factors were reef type (dead margin or reference; fixed), site nested within reef type (random), and month (fixed).

\begin{tabular}{lrccr}
\hline \hline \multicolumn{1}{c}{ Source } & df & Mean Square & F & Significance \\
\hline Reef type & 1 & 0.003 & 0.000 & 0.985 \\
Site (Reef type) & 4 & 8.019 & 1.574 & 0.181 \\
Month & 11 & 37.699 & 9.340 & $<0.001$ \\
Residual & 343 & & & \\
\hline
\end{tabular}

Tolley et al. 2005, Tolley \& Volety 2005). Our data also support earlier research conducted in the Indian River Lagoon system that looked at the sessile species diversity on hard substrata, although not specifically associated with Crassostrea virginica (Mook 1976, 1980, 1981 and 1983). As is typical for shellfish assemblages (O'Beirn et al. 2004), the oyster reef community within Mosquito Lagoon was dominated (in terms of abundance) by only a few taxa (i.e., Annelida, Arthropoda and Chordata) (Table 1 and 5).

\section{Sessile Macrofauna}

The most abundant sessile species in the nets were in the genus Balanus. These organisms were present year-round on oyster reefs and numerically dominant in all nets. Balanus eberneus, the native ivory barnacle recruited to shells placed in lift nets during each month of our survey (Table 1). Monthly recruitment ranged from 87 recruits in February 2005 to 2,447 in July 2004. In fact, every net always had at least one B. eberneus. Balanus amphitrite, the purple striped barnacle, invaded the IRL approximately $100 \mathrm{y}$ ago (J. Carlton pers. comm.). It was common but not as abundant as its conger (Table 1). Numbers of B. amphitrite decreased dramatically during the colder months of the year (Table 1). The abundance of these barnacles in this system outcompeting $C$. virginica for space may be associated with declines in oyster populations in Mosquito Lagoon (Boudreaux 2005). Dense sets of Balanus spp. monopolizing all free space on oyster reefs suggest intense spatial competition between oysters and barnacles in the IRL during summer and fall months (Boudreaux 2005).

The nonnative bivalve mussel, Mytella charruana, was found during this study (Boudreaux \& Walters 2006). This South American bivalve was first found in lift nets in August 2004 and has since rapidly spread within northern Mosquito Lagoon (Boudreaux $\&$ Walters 2006). Although low numbers of this species may have predated our study, no individuals were recorded in a 3-y study in these waters between 1998 to 2001 (L. Walters unpublished data).

\section{TABLE 7.}

Three-factor nested ANOVA comparing density of motile species in lift nets. Factors were reef type (dead margin or reference; fixed), site nested within reef type (random), and month (fixed).

\begin{tabular}{lrrrr}
\hline \hline \multicolumn{1}{c}{ Source } & df & Mean Square & \multicolumn{1}{c}{ F } & Significance \\
\hline Reef type & 1 & 356.001 & 0.281 & 0.624 \\
Site (Reef type) & 4 & 1267.778 & 4.430 & 0.002 \\
Month & 11 & 2379.484 & 10.278 & $<0.001$ \\
Residual & 343 & & & \\
\hline
\end{tabular}




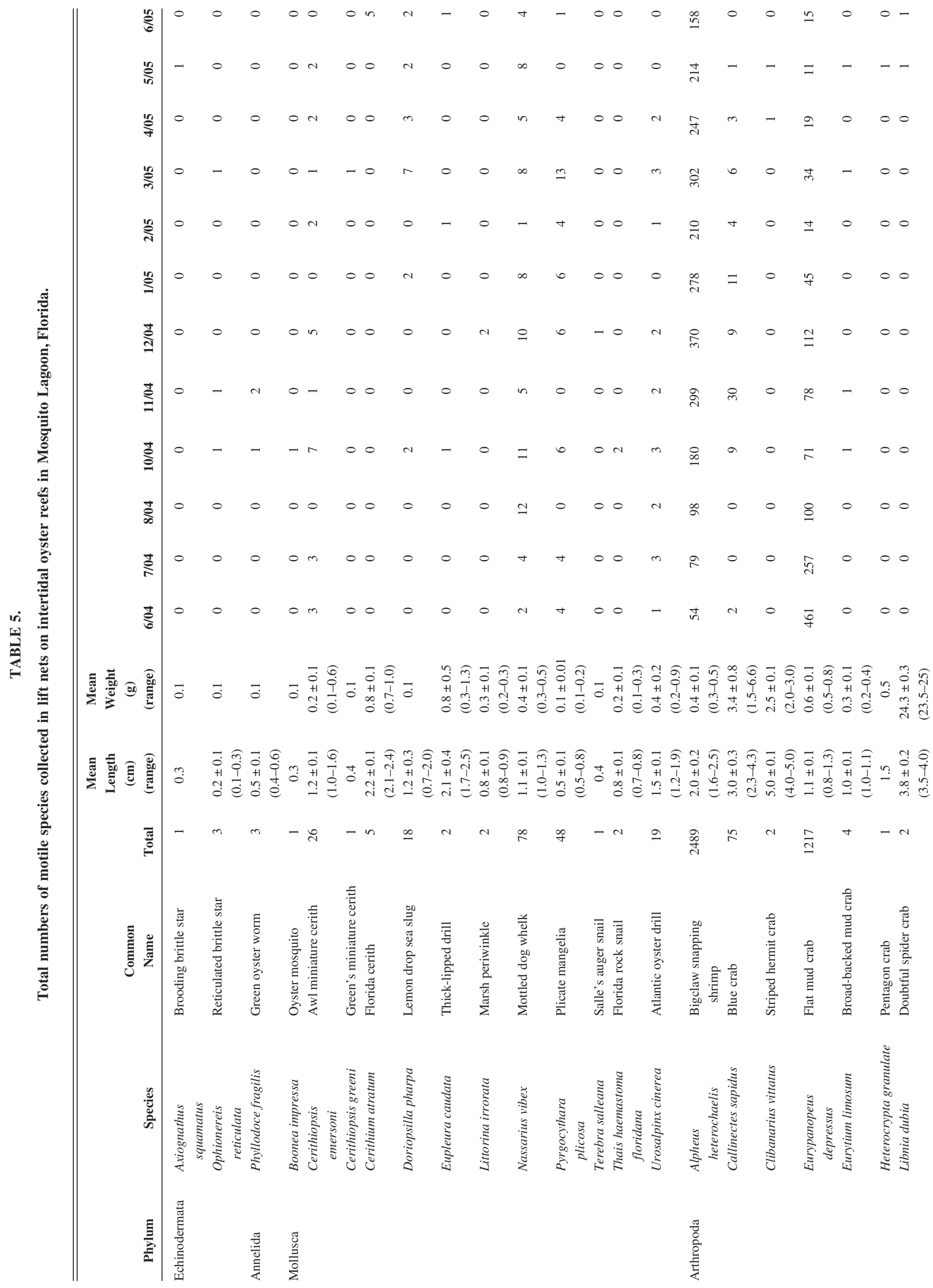




\begin{tabular}{|c|c|c|c|c|c|c|c|c|c|c|c|c|c|c|c|c|c|c|c|c|c|}
\hline 0 & ले & $q$ & $\stackrel{\infty}{\sim}$ & $a$ & i̊ & $-d$ & - & $0-$ & 0 & 000 & $r$ & ते & $\infty$ & $m$ & 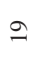 & $=$ & 0 & 0 & $0 \infty$ & 000 & 2 \\
\hline 0 & F & $m$ & in & t & 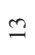 & $\circ \mathrm{m}$ & 0 & 00 & 0 & 000 & 0 & लै & $m$ & in & ㄱ. & 于 & $m$ & 0 & $0 \infty$ & 000 & $m$ \\
\hline$N$ & $\stackrel{\infty}{-}$ & $\infty$ & 우 & $a$ & 2 & 00 & 0 & 00 & 0 & 000 & $N$ & $\infty$ & $\simeq$ & 0 & ฉે & t & - & 0 & o $n$ & -00 & 0 \\
\hline 0 & 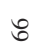 & $\stackrel{\infty}{+}$ & 0 & \pm & in & 00 & 0 & 00 & 0 & 000 & - & in & + & 0 & 요 & in & $N$ & 0 & $\circ \mathrm{m}$ & $0-0$ & 0 \\
\hline 0 & $\alpha$ & $\bar{\sim}$ & 0 & 6 & $\simeq$ & 00 & 0 & 00 & 0 & $00-$ & 0 & チ & $m$ & 0 & 9 & 0 & 0 & 0 & 00 & $00 \mathrm{~N}$ & 0 \\
\hline 0 & in & F & in & $\stackrel{\circ}{\sim}$ & $=$ & $0+$ & 0 & 00 & 0 & 000 & $m$ & $\widehat{\sigma}$ & 0 & 0 & फे & $=$ & 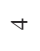 & 0 & $0-$ & 000 & 0 \\
\hline 0 & $\vec{i}$ & के & 0 & ల & 우 & 00 & 0 & -- & - & 000 & $m$ & $\stackrel{\infty}{\sim}$ & $\hat{\imath}$ & 0 & 0 & - & $N$ & - & -0 & $00 \pi$ & 0 \\
\hline- & $\stackrel{\sim}{\sim}$ & $\stackrel{\infty}{\infty}$ & $m$ & $\cong$ & $=$ & 00 & 0 & $\circ \mathrm{m}$ & - & $00-$ & 2 & $\underline{6}$ & ले & 0 & - & $n$ & $ㅇ$ & 0 & o $n$ & $00 \cdots$ & 0 \\
\hline 0 & \pm & A & in & $a$ & i & or & $m$ & $0-$ & 0 & 000 & $\stackrel{\infty}{\sim}$ & $\vec{m}$ & $\stackrel{f}{f}$ & 0 & 0 & 0 & - & 0 & 00 & 000 & 0 \\
\hline 0 & $N$ & $\bar{n}$ & $a$ & ते & $=$ & $0-$ & 0 & 00 & 0 & -00 & - & $\stackrel{d}{d}$ & $\simeq$ & 0 & $N$ & 0 & - & - & o $n$ & $00 \mathrm{~N}$ & 0 \\
\hline- & 0 & $\frac{1}{4}$ & $m$ & in & 0 & $0-$ & 0 & 00 & 0 & $0-0$ & 0 & $\tilde{\lambda}$ & $m$ & 0 & - & 0 & 0 & 0 & $0-$ & 000 & 0 \\
\hline 0 & - & R & 0 & $\infty$ & 0 & om & $m$ & 00 & - & 000 & 0 & 9 & - & 0 & $m$ & 0 & - & 0 & $0+$ & $00-$ & 0 \\
\hline
\end{tabular}

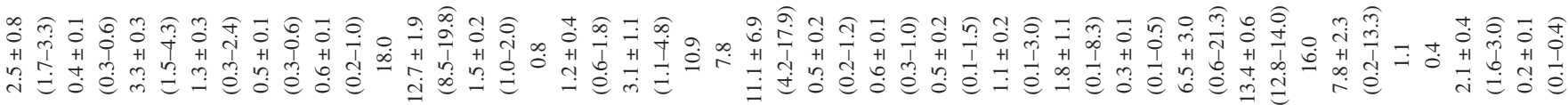

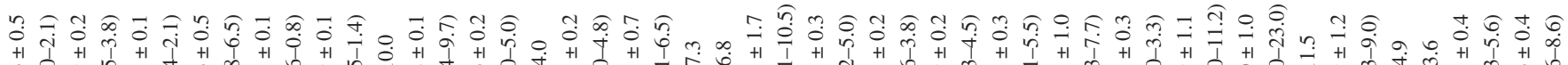

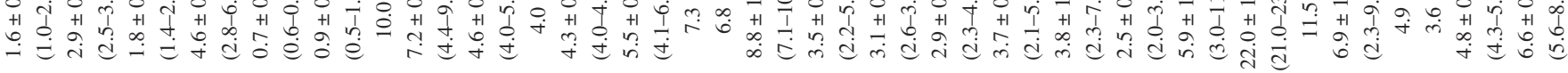

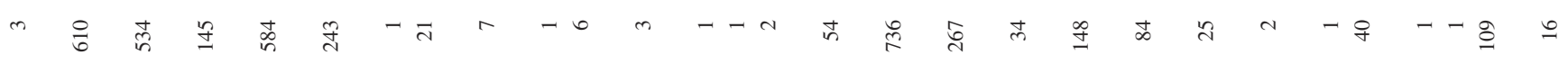

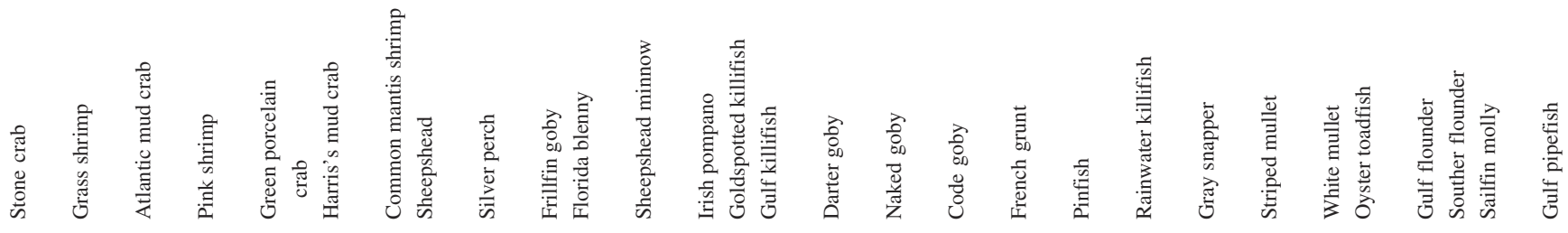

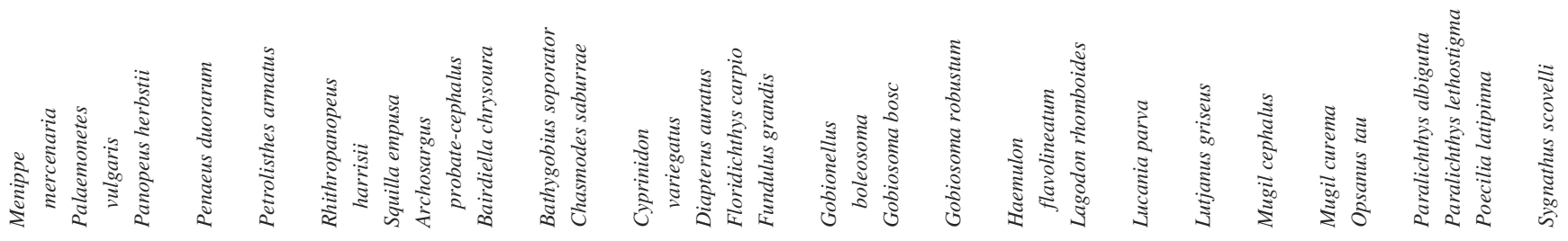



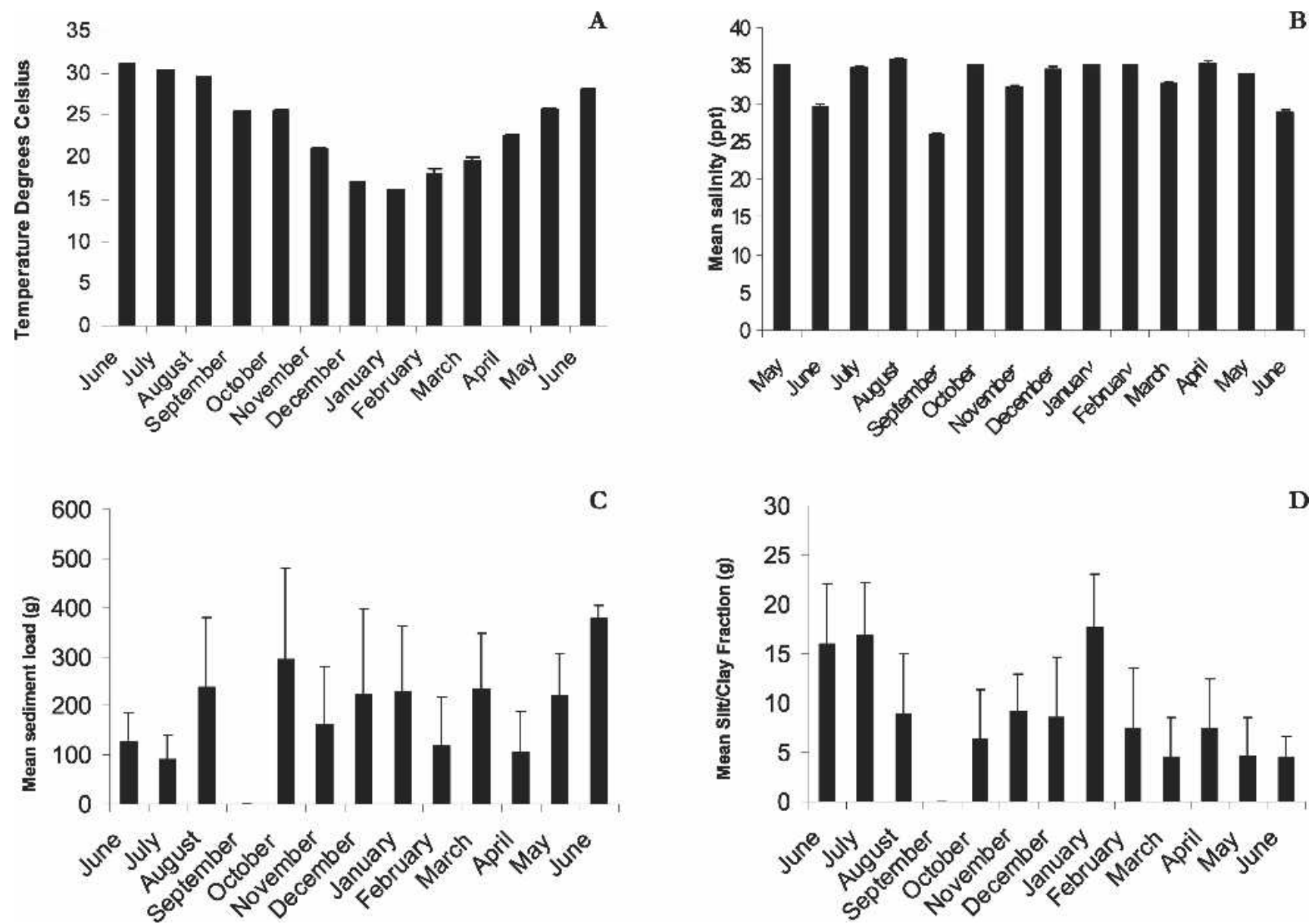

Figure 5. Abiotic variables (a). Mean monthly temperatures $( \pm \mathrm{SE})$ at lift net sampling dates in Mosquito Lagoon. (b) Mean salinities ( \pm SE) of Mosquito Lagoon on lift net sampling dates. (c) Mean sediment load $( \pm \mathrm{SE}$ ) per month from June 2004 to June 2005. (d) Mean silt/clay percentage $( \pm$ SE) per month from June 2004 to June 2005.

One individual of the invasive Asian green mussel Perna viridis, which has devastated some oyster reefs in Tampa Bay, Florida (Baker et al. 2003), was also recently found on a piling in Mosquito Lagoon (MB pers. obs.). It has not been found on Mosquito Lagoon oyster reefs to date. Both nonnative bivalves continue to be monitored within the IRL.

\section{Motile Macrofauna}

The two most abundant motile species sampled within the lift nets were the bigclaw snapping shrimp Alpheus heterochaelis

\section{TABLE 8.}

Three-factor nested ANOVA comparing total sediment loads collected per month at lift net sites. Factors were reef type (dead margin or reference; fixed), site nested within reef type (random), and month (fixed).

\begin{tabular}{lrccc}
\hline \hline \multicolumn{1}{c}{ Source } & df & Mean Square & F & Significance \\
\hline Reef type & 1 & 317305.223 & 1.961 & 0.234 \\
Site (Reef type) & 4 & 161861.650 & 3.357 & 0.011 \\
Month & 11 & 124938.063 & 2.624 & 0.004 \\
Residual & 199 & & & \\
\hline
\end{tabular}

(2,489 individuals) and the flat mud crab Eurypanopeus depressus (1,217 individuals) (Table 5). Previous studies found these two species to be present in temperate waters on both the Atlantic coast of North Carolina (132 individuals of E. depressus; Glancy et al. $2003)$ and the gulf coast of Florida (3,184 individuals of $E$. depressus, 364 individuals of A. heterochaelis; Meyer 1994). Similar to Tolley et al. (2005), we found the replacement of temperate species by tropical cogeners, including the replacement of the striped blenny Chasmodes bosquianus in the Northern Atlantic (Breitburg 1999, Coen et al. 1999) by the Florida blenny Chasmodes saburrae.

TABLE 9.

Three-factor nested ANOVA comparing silt/clay fractions collected per month at lift net sites. Factors were reef type (dead margin or reference; fixed), site nested within reef type (random), and month (fixed).

\begin{tabular}{lrccr}
\hline \hline \multicolumn{1}{c}{ Source } & df & Mean Square & F & Significance \\
\hline Reef type & 1 & 39.068 & 0.687 & 0.454 \\
Site (Reef type) & 4 & 56.862 & 0.871 & 0.482 \\
Month & 11 & 409.454 & 9.004 & $<0.001$ \\
Residual & 199 & & & \\
\hline
\end{tabular}


The fifth most abundant mobile species was the green porcelain crab, Petrolisthes armatus (Table 5). It is considered an invasive exotic along the South Atlantic Bight (Knott et al. 1999, Glancy et al. 2003). Populations of this species can historically be found in the Pacific (i.e., California to Peru) and the Atlantic (i.e., Africa, Ascension Island, Bermuda, Bahamas, Gulf of Mexico, West Indies, Caribbean and South America down to Brazil; Knott et al. 1999). Although the pathway of introduction remains unknown, ballast transport and increasing winter temperatures, which favor its establishment are possibilities (Knott et al. 1999). It was first collected along Florida's east coast in the 1930s in Biscayne Bay and Miami Beach (Knott et al. 1999). Slowly, it spread northward, becoming well established in the Indian River Lagoon system (Knott et al. 1999). Studies have shown abundances to increase dramatically in only a few years after introduction (Knott et al. 1999). The current range of $P$. armatus along the South Atlantic Bight stretches from South Carolina down to the southern tip of Florida (Knott et al. 1999).

Size and biomass data for several crustacean and fish species revealed that both juvenile and adult individuals were present on the reefs (Table 5). For example, large ranges were seen for the big-claw snapping shrimp Alpheus heterochaelis (length 1.6-2.5 $\mathrm{cm}$; biomass $0.3-0.5 \mathrm{~g}$ ), blue crab Callinectes sapidus (length 2.3-4.3 cm; biomass 1.5-6.6 g), stone crab Menippe mercenaria (length 1.0-2.1 cm; biomass 1.7-3.3 g), grass shrimp Palaemonetes vulgaris (length $2.5-3.8 \mathrm{~cm}$, biomass $0.3-0.6 \mathrm{~g}$ ) and pink shrimp Penaeus duorarum (length $2.8-6.5 \mathrm{~cm}$, biomass $0.3-2.4 \mathrm{~g}$ ) (Table 5). Within the Chordata family, different life stages were seen for the sheepshead Archosargus probatocephalus (length 4.4$9.7 \mathrm{~cm}$, biomass 8.5-19.8 g), pinfish Lagodon rhomboides (length 2.3-7.7 cm, biomass 0.1-8.3), gray snapper Lutjanus griseus (length 3.0-11.2 cm, biomass 0.6-21.3 g), oyster toadfish Opsanus tau (length 2.3-9.0 cm; biomass 0.2-13.3 g), and numerous killifish and goby species (Table 5).

The blue crab Callinectes sapidus, the pink shrimp Penaeus duorarum and juvenile forms of several important finfish species were collected in the lift nets within Mosquito Lagoon (Table 5). Hence, commercially and recreationally valuable species are utilizing oyster reefs within Mosquito Lagoon, confirming the importance of oyster reefs to the economy of this region. These species were also found to be utilizing oyster reefs on the west coast of Florida (Tolley et al. 2005).

Additional comparisons can be made with the motile species found on oyster reefs in Mosquito Lagoon to lift net studies of intertidal reefs on the west coast of Florida $\left(26^{\circ} 25^{\prime} 56^{\prime \prime} \mathrm{N}\right.$, $81^{\circ} 48^{\prime} 34^{\prime \prime} \mathrm{W}$ ) (Tolley et al. 2005, Tolley \& Volety 2005). Salinities and temperatures found in Mosquito Lagoon (mean: $33 \mathrm{ppt}$, $23.8^{\circ} \mathrm{C}$ ) are comparable to the system studied in southwest Florida (mean: $32.5 \mathrm{ppt}, 27.1^{\circ} \mathrm{C}$ ). Overall species richness (the total number of species found per net) was found to be similar between the two different Florida locations (Gulf: 4-11 species/month versus Mosquito Lagoon: 4-11 species/month), whereas density was slightly lower in Mosquito Lagoon (Gulf: 20-400 organisms/net versus Mosquito Lagoon: 12-40 organisms/net). In both systems, there were more fishes than decapod crustacean species (Gulf: 16 versus 9, Mosquito Lagoon: 23 versus 18). In both locations, decapod crustaceans dominated all motile samples numerically.

\section{Dead Margins Affect on Oyster Reef Communities}

Dead margins, attributed to wakes from recreational boating in Mosquito Lagoon (Grizzle et al. 2002, Wall et al. 2005), did not have a significant effect on the back-reef usage of oysters as substrate by either sessile or motile species (Tables 3, 4, 6 and 7). The back-reef areas of both were also visually very similar. This suggests the back-reef areas on oyster reefs with dead margins function similarly to a reference oyster reef with no dead margin.

Although dead margins did not have an impact on richness or density of organisms found, sessile organisms preferred to settle on living oyster clumps rather than on the disarticulated shells placed within the nets (Fig. 4). The 3-dimensional structure of the two settlement substrates was very different. Disarticulated shells were single and loose, laid flat on the benthos, and were often covered by sediment. These shells were frequently displaced by water motion. Live oysters attached together to form clusters and rarely moved. These 3 -dimensional clusters probably provided more protection and refuge from predators for sessile inhabitants than the 2-dimensional, disarticulated shells. Subsequent research has shown that reference reefs contained twice more oyster clumps than reefs with dead margins within Mosquito Lagoon (Stiner 2006). Combined, these results reveal a new negative impact of dead margins on sustaining biodiversity in Mosquito Lagoon.

Wall et al. (2005) found an increase in sediment accumulation on the seaward edges (fore-reef areas) of reefs with dead margins in Mosquito Lagoon and suggested this was caused by sediment resuspension associated with large numbers of boat wakes. Increased sediment has been shown to decrease the settlement of Crassostrea virginica (Boudreaux 2005). Thus, any difference in sediment loads between locations would have been predicted to have an effect on species assemblages between the two types of reefs (reference and dead margins). However, this study focused exclusively on the back-reef regions of oyster reefs and did not show any differences in sediment loads between reef types. Dead margins are hypothesized to protect these back-reef areas by preventing sediment accumulation.

During this study we documented the usage of intertidal oyster reefs in Mosquito Lagoon by 105 different species. This included 76 invertebrates and 29 chordates. The richness in diversity found within the reefs of Crassostrea virginica is comparable with other systems in the Indian River Lagoon system. A study of decapods associated with seagrass communities in the Indian River Lagoon showed remarkable diversity. In all, 38 decapod species were found in seagrass beds (Gore et al. 1981; Smithsonian Institution 2006) as compared with the 19 decapod species we found using oyster reefs (Tables 2 and 5). These examples demonstrate the extremely high diversity in the IRL that can be attributed to its important habitats, including seagrass beds and oyster reefs. The data from this study are an important step to gaining a better understanding of these oyster reefs and their essential role in the estuary. Additionally, this data provides a baseline from which to evaluate efforts to practice sustainable ecosystem management of Crassostrea virginica within Mosquito Lagoon.

\section{ACKNOWLEDGMENTS}

The authors thank the University of Central Florida and Canaveral National Seashore for their support in this project. Funding was provided by the University of Central Florida Biology Department, including Research Enhancement Grants (MB, JS), as well as the Aylesworth Foundation (JS), Sigma Xi Grant in Aid of Research (MB), and Florida Sea Grant (LW). We greatly appreciate all those who helped with data collection: M. Black, M. 
Donnelly, N. Gillis, C. Glardon, J. Ledgard, A. McLellan, J. Sacks, P. Sacks, A. Simpson, E. Stiner, J. C. Stiner, and J. K. Stiner. We thank A. Benson, R. Gilmore, L. Haynes, C. Holden, H. Lee, and E. Reyier for help with species identification and A. Volety, G.
Tolley and L. Coen for help with the methodology. Finally, we thank I. J. Stout, M. Luckenbach, L. Morris, R. Noss, P. Quintana, J. Fauth and an anonymous reviewer for greatly improving the text and analyses.

\section{LITERATURE CITED}

Bahr, L. M. \& W. P. Lanier. 1981. The Ecology of Intertidal Oyster Reefs of the South Atlantic Coast: A Community Profile. Fish and Wildlife Service, Office of Biological Services Report FWS/OBS-81/15. $105 \mathrm{pp}$.

Baker, P., J. Fajans \& D. Bergquist. 2003. Invasive Green Mussels, Perna viridis, on Mangroves and Oyster Reefs in Florida, University of Florida. Proceedings of the Third International Conference on Marine Bioinvasions, La Jolla, California, March 16-19, 2003. pp. 10.

Bartol, I. K., R. Mann \& M. Luckenbach. 1999. Growth and mortality of oyster (Crassostrea virginica) on constructed intertidal reefs. J. Exp. Mar. Biol. Ecol 237:157-184.

Boudreaux, M. 2005. Native and invasive sessile competitors of the eastern oyster Crassostrea virginica in Mosquito Lagoon, Florida. University of Central Florida, Orlando Florida: M.S. Thesis. 104 pp.

Boudreaux, M. L. \& L. J. Walters. 2006. Mytella charruana: A New, Invasive Bivalve in Mosquito Lagoon, Florida. Nautilis 120:34-36.

Breitburg, D. L. 1999. Are three-dimensional structure and healthy oyster populations the keys to an ecologically interesting and important fish community? In: M. W. Luckenbach, R. Mann \& J. A. Wesson, editors. Oyster reef habitat restoration: a synopsis and synthesis of approaches. Gloucester Point, Virginia, Virginia Institute of Marine Science Press. pp. 239-250.

Coen, L. D., D. M. Knott, E. L. Wenner, N. H. Hadly, A. H. Ringwold \& M. Y. Bobo. 1996a. Intertidal oyster reef studies in South Carolina: design, sampling and experimental focus for evaluating habitat value and function. In: M.W. Luckenbach, R. Mann and J. A. Wesson, editors. Oyster reef habitat restoration: a synopsis and synthesis of approaches. Gloucester Point, Virginia. Virginia Institute of Marine Science Press. pp. 239-250.

Coen, L., M. Luckenbach \& D. L. Breitburg. 1999. The role of oyster reefs as essential fish habitat: A review of current knowledge and some new perspectives. Amer. Fish. Society Symposium 22:438-454.

Coen, L. D., E. L. Wenner, D. M. Knott, B. W. Stender, N. H. Hadley \& M. Y. Bobo. 1996b. Intertidal oyster reefs as critical estuarine environments: evaluating habitat use, development and function. J. Shellfish Res. 15:490.

Crabtree, R. E. \& J. M. Dean. 1982. The structure of two South Carolina estuarine tide pool fish assemblages. Estuaries 5:2-9.

Dame, R. F. 1979. The abundance, diversity, and biomass of macrobenthos on North Inlet, South Carolina, intertidal oyster reefs. Proc. Natl. Shellfish Assoc 69:6-10.

Dame, R. F. 1996. Ecology of marine bivalves: ecosystems approach. CRC Marine Science Series, Boca Raton, Florida, USA. 256 pp.

Dawes, C. J., D. Hanisak \& W. J. Kenworthy. 1995. Seagrass biodiversity in the Indian River Lagoon. Bull. Mar. Sci. 57:59-66.

Gilmore, R. G. 1977. Fishes of the Indian River lagoon and adjacent waters, Florida. Bulletin of the Florida State Museum 22:101-147.

Gilmore, R. G. 1995. Environmental and biogeographic factors influencing ichthyofaunal diversity: Indian River Lagoon. Bull. Mar. Sci. 57:153170.

Glancy, T. P., T. K. Frazer, C. E. Cichra \& W. J. Lindberg. 2003. Comparative patterns of occupancy by decapod crustaceans in seagrass, oyster, and marsh-edge habitats in a Northeast Gulf of Mexico estuary. Estuaries 26:1291-1301.

Gore, R. H., E. E. Gallaher, L. E. Scotto \& K. A. Wilson. 1981. Studies on decapod crustacea from the Indian River region of Florida. Estuar. Coast. Shelf Sci. 12:485-508.
Grizzle, R. E. 1990. Distribution and abundance of Crassostrea virginica (Gmelin, 1791) (eastern oyster) and Mercenaria spp. (quahogs) in a coastal lagoon. J. Shellfish Res. 9:347-358.

Grizzle, R. E., J. R. Adams \& L. J. Walters. 2002. Historical changes in intertidal oyster (Crassostrea virginica) reefs in a Florida lagoon potentially related to boating activities. J. Shellfish Res. 2:749-756.

Jackson, J. C., M. X. Kirby, W. H. Berger, K. A. Bjorndal, L. W. Botsford, B. J. Bourque, R. H. Bradbury, R. Cooke, J. Erlandson, J. A. Estes, T. P. Hughes, S. Kidwell, C. B. Lange, H. S. Lenihan, J. M. Pandol, C. H. Peterson, R. S. Steneck, M. J. Tegner \& R. R. Warner. 2001. Historical overfishing and the recent collapse of coastal ecosystems. Science 293:629-639.

Knott, D., C. Boyko \& A. Harvey. 1999. Introduction of the green porcelain crab, Petrolisthes armatus (Gibbes, 1850) into the South Atlantic Bight. In: J. Pederson, editor. Marine Bioinvasions: the Proceedings of the First National Conference. Massachusetts Institute of Technology, Cambridge, Massachusetts. pp. 404.

Lenihan, H. S. 1999. Physical-biological coupling on oyster reefs: how habitat structure influences individual performance. Ecol. Monog. 69: 251-276.

Micheli, F. \& C. H. Peterson. 1999. Estuarine vegetative habitats as corridors for predator movement. Con. Bio. 13:869-881.

Mook, D. H. 1976. Studies on fouling invertebrates in the Indian River. I. Seasonality of settlement. Bull. Mar. Sci. 26:610-615.

Mook, D. H. 1980. Seasonal variation in species composition of recently settled fouling communities along an environmental gradient in the Indian River Lagoon, Florida. Estuar. Coast. Shelf Sci. 2:573-581.

Mook, D. H. 1981. Effects of disturbance and initial settlement on fouling community structure. Ecology 62:522-526.

Mook, D. H. 1983. Indian River Lagoon fouling organisms, a review. Florida Scientist 46:162-167.

Meyer, D. L. 1994. Habitat partitioning between the Xanthid crabs Panopeus herbstii and Eurypanopeus depressus on intertidal oyster reefs (Crassostrea virginica) in southeastern North Carolina. Estuaries 17: 674-679.

O'Beirn, F. X., P. G. Ross \& M. W. Luckenbach. 2004. Organisms associated with oysters cultured in floating systems in Virginia, USA. J. Shellfish Res. 23:825-829.

Posey, M. H., T. D. Alphin \& C. M. Powell. 1999. Use of oyster reef as habitat for epibenthic fish and decapods. In: M. W. Luckenbach, R. Mann \& J. A. Wesson, editors. Oyster reef habitat restoration: a synopsis and synthesis of approaches. Gloucester Point, Virginia. Virginia Institute of Marine Science Press. pp. 239-250.

Provancha, J. A., C. R. Hall \& D. M. Oddy. 1992. Mosquito Lagoon environmental resources inventory. NASA Technical Memorandum 107548. The Bionetics Corporation. Kennedy Space Center, Florida. $111 \mathrm{pp}$.

Smithsonian Institution 2006. Indian River Lagoon Species Inventory Report. Website: http://www.sms.si.edu/irlspec/index.htm.

Snelson, F. F., Jr. \& S. E. Williams. 1981. Notes on the occurrence, distribution, and biology of elasmobranch fishes in the Indian River Lagoon system, Florida. Estuaries 4:110-120.

Stiner, J. 2006. Predation on the eastern oyster Crassostrea virginica on intertidal reefs affected by recreational boating. University of Central Florida, Orlando, Florida: M.S. Thesis. 114 pp.

Tolley, S. G. \& A. K. Volety. 2005. The role of oysters in habitat use of oyster reefs by resident fishes and decapod crustaceans. J. Shellfish Res. 24:1007-1012.

Tolley, S. G., A. K. Volety \& M. Savarese. 2005. Influence of salinity on 
the habitat use of oyster reefs in three southwest Florida estuaries. J. Shellfish Res. 24:127-138.

Tremain, D. M. \& D. H. Adams. 1995. Seasonal variations in species diversity, abundance, and composition of fish communities in the northern Indian River Lagoon, Florida. Bull. Mar. Sci. 57:171-192.

Virnstein, R. W., P. S. M. Ickkelson, K. D. Cairns \& M. A. Capone. 1983. Seagrass beds versus sand bottoms: the trophic importance of their associated benthic invertebrates. Florida Scientist 46:363-381.

Wall, L. M., L. J. Walters, R. E. Grizzle \& P. E. Sacks. 2005. Recreational boating activity and its impacts on the recruitment and survival of the oyster Crassostrea virginica on intertidal reefs in Mosquito Lagoon, Florida. J. Shellfish Res. 24:965-974.

Walters, L., A. Roman, J. Stiner \& D. Weeks. 2001. Water Resource Management Plan, Canaveral National Seashore. Titusville, Florida, National Park Service, Canaveral National Seashore. 224 pp.

Wells, H. W. 1961. The fauna of oyster reefs with special reference to the salinity factor. Ecol. Monogr. 60:449-469.

Wenner, E., H. R. Beatty \& L. Coen. 1996. A quantitative system for sampling nekton on intertidal oyster reefs. J. Shellfish Res. 15:769-775. 